\title{
Coherent dynamics of plasma mirrors
}

\author{
C. THAURY' ${ }^{1}$, H. GEORGE 1 , F. QUÉRÉ ${ }^{1 *}$, R. LOCH ${ }^{2}$, J.-P. GEINDRE ${ }^{3}$, P. MONOT ${ }^{1}$ AND PH. MARTIN ${ }^{1}$ \\ ${ }^{1}$ CEA, IRAMIS, Service des Photons Atomes et Molécules, F-91191 Gif-sur-Yvette, France \\ ${ }^{2}$ Laser Physics and Nonlinear Optics Group, Faculty of Science and Technology, MESA ${ }^{+}$Institute for Nanotechnology, University of Twente, The Netherlands \\ ${ }^{3}$ Laboratoire pour I'Utilisation des Lasers Intenses, CNRS, Ecole Polytechnique, 91128 Palaiseau, France \\ *e-mail: fabien.quere@cea.fr
}

Coherent ultrashort $X$-ray pulses provide new ways to probe matter and its ultrafast dynamics ${ }^{1-3}$. One of the promising paths to generate these pulses consists of using a nonlinear interaction with a system to strongly and periodically distort the waveform of intense laser fields, and thus produce high-order harmonics. Such distortions have so far been induced by using the nonlinear polarizability of atoms, leading to the production of attosecond light bursts ${ }^{4}$, short enough to study the dynamics of electrons in matter ${ }^{3}$. Shorter and more intense attosecond pulses, together with higher harmonic orders, are expected ${ }^{5,6}$ by reflecting ultraintense laser pulses on a plasma mirror-a dense $\left(\approx 10^{23}\right.$ electrons $\left.\mathrm{cm}^{-3}\right)$ plasma with a steep interface. However, short-wavelength-light sources produced by such plasmas are known to generally be incoherent ${ }^{7}$. In contrast, we demonstrate that like in usual low-intensity reflection, the coherence of the light wave is preserved during harmonic generation on plasma mirrors. We then exploit this coherence for interferometric measurements and thus carry out a first study of the laser-driven coherent dynamics of the plasma electrons.

One of the challenges of high-order harmonic generation (HHG), beyond the production of very high orders with good efficiencies, is to preserve the initial properties of the laser beam in this frequency conversion process. In particular, keeping a high degree of coherence is essential for many applications, such as coherent imaging ${ }^{8}$. This has already been shown to be possible for HHG in gases ${ }^{9}$, but remains an open question for dense plasmas.

In this case, an intense laser pulse interacts with an initially solid target, and creates a dense reflective plasma at the surface. In an early study using picosecond laser pulses ${ }^{10}$, interference measurements in the far-field using Young slits suggested that the coherence of the light field in the source plane was lost in interaction with such an extremely dense and hot (a few $10^{6} \mathrm{~K}$ ) plasma, for instance through stochastic processes or plasma instabilities. This conclusion was consistent with the observation of an uncollimated harmonic emission. Here, we demonstrate that such deleterious effects can be avoided using well-controlled interaction conditions, that is, ultrashort $(<100 \mathrm{fs})$ laser pulses with a high temporal contrast. In these conditions, the plasma hardly has time to expand during the interaction (density gradient scale length $L \ll \lambda / 10$, with $\lambda$ being the laser wavelength), and thus behaves as a high-flatness mirror-a plasma mirror ${ }^{11}$. We then exploit the coherence of the produced harmonics to study the dynamics of plasma electrons on the attosecond timescale.

Our experiment uses $60 \mathrm{fs}$ pulses with a high temporal contrast $\left(10^{10}\right.$ on the nanosecond timescale), to produce high-order harmonics on plasma mirrors through coherent wake emission ${ }^{12}$ (CWE), at intensities from a few $10^{16} \mathrm{~W} \mathrm{~cm}^{-2}$ to a few $10^{17} \mathrm{~W} \mathrm{~cm}^{-2}$.
Groups of harmonics in the beam diverging from the plasma mirror are selected with different kinds of thin metallic filter, and the resulting spatial profile in the far-field is then measured at a distance $D=38 \mathrm{~cm}$ from the source (see the Methods section). It has already been shown that collimated beams of extreme-ultraviolet light are obtained in such an interaction regime ${ }^{11}$, but this result alone does not guarantee a good spatial coherence of the harmonics ${ }^{13}$.

We have implemented a new and remarkably simple technique to study this issue. A transmission grating is placed into the beam before focusing (Fig. 1a). It is designed in such a way that at the focus of the beam, diffraction produces a central focal spot surrounded by two slightly weaker satellite spots at a distance $a=40 \mu \mathrm{m}$. Temporally synchronized and phase-locked laser pulses reach these three spatially separated foci of identical shape. Highorder harmonics are generated on each focal spot on reflection onto a plasma mirror.

When measuring the spatial profile of the harmonic beam in the far-field, fringes with an almost perfect contrast can be observed, which result from the interferences between the three spatially separated extreme-ultraviolet sources (Fig. 1b,c). This simple result demonstrates the mutual coherence of several harmonic sources generated on a plasma mirror. It establishes that, although it involves a highly complex medium, the HHG mechanism is coherent, that is, it preserves the phase properties of the incident light. This is, to the best of our knowledge, the first direct proof of the intrinsic coherence of a frequency up-conversion process in such a dense plasma. Such mutually coherent short-wavelength sources can be exploited in applications such as time-resolved interferometry ${ }^{14}$, holography or high-resolution Ramsey spectroscopy ${ }^{15}$. This essential property adds to the recent surge of results obtained on this light source ${ }^{5,6,11,16,17}$, showing that plasma mirrors are a very promising path to coherent ultrashort and intense X-ray pulses.

These measurements involve the interference of several harmonic sources driven at different laser intensities. Changes in laser intensity $I$ imply variations in the dynamics of the system, and hence in the temporal properties of the radiated harmonic field. In particular, the spectral phase $\phi_{\omega}$ of the harmonics generally depends on laser intensity ${ }^{18-20}$. Interferograms such as the one shown in Fig. 1b can provide information on $\phi_{\omega}(I)$, and hence on the laser-driven coherent dynamics of the plasma electrons. In addition, knowledge of this intensity-dependent phase is essential to understand the properties of any laser-driven harmonic source. Indeed, because high laser intensities are required, HHG occurs around the focus of ultrashort laser pulses, and the laser intensity thus has strong spatial and temporal variations. These lead to non-trivial spatial and temporal phases of the harmonics, which 

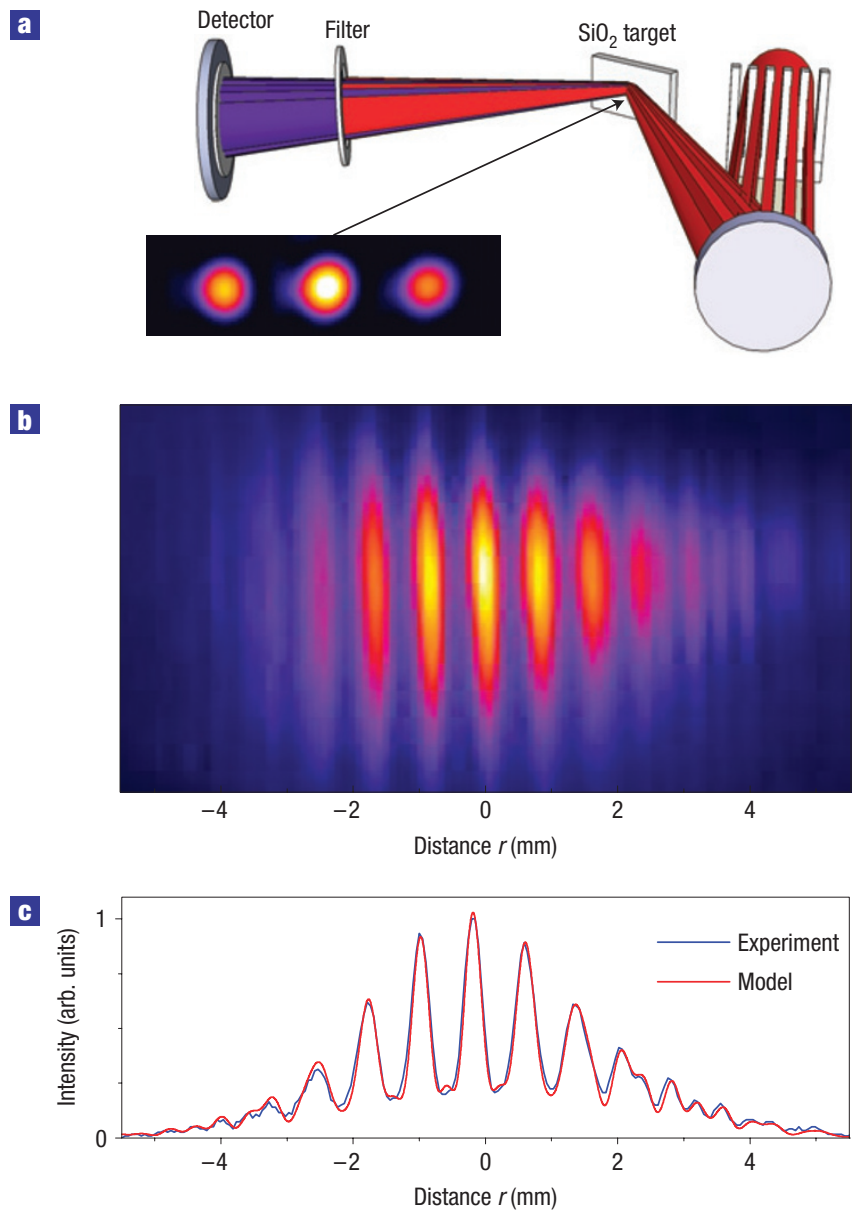

Figure 1 Coherent harmonic beams from plasma mirrors. a, Experimental set-up, and intensity distribution in the focal plane. In this case, the intensity ratio between the central and lateral spots is 0.57 . $\mathbf{b}$, Single-shot far-field interference pattern of harmonics 8-10. c, Lineout of the fringes and theoretical fit with phase shifts $\Delta \Phi_{\omega}=0.23 \omega / \omega_{\mathrm{L}}$ rad.

affect the divergence and spectral width, as well as the exact coherence degree of the source ${ }^{20,21}$.

In the case of three sources of different intensities shown in Fig. 1a, the interference pattern in the far-field is given by the following elementary equation (see the Supplementary Information)

$$
\begin{gathered}
I(\mathbf{r})=\int \mathrm{d} \omega I_{\omega} F_{\omega}(\mathbf{r})\left[I_{\omega}^{0}+I_{\omega}^{1}(\mathbf{r})+I_{\omega}^{2}(\mathbf{r})\right] \\
I_{\omega}^{0}=1+2 \alpha_{\omega} \\
I_{\omega}^{1}(\mathbf{r})=4 \sqrt{\alpha_{\omega}} \cos \left(\Delta \phi_{\omega}\right) \cos \left(\mathbf{k}_{\omega} \mathbf{r}\right) \\
I_{\omega}^{2}(\mathbf{r})=2 \alpha_{\omega} \cos \left(2 \mathbf{k}_{\omega} \mathbf{r}\right),
\end{gathered}
$$

where $\mathbf{k}_{\omega}=(\omega / c D) \mathbf{a}$ (with $c$ being the speed of light and $\mathbf{a}$ being the vector joining the central focal spot to a lateral one), $I_{\omega}$ is the harmonic spectral intensity and $F_{\omega}(\mathbf{r})$ is the spatial intensity profile of frequency $\omega$ in the detection plane for a single focal spot. $\alpha_{\omega}$ is the intensity ratio between the central harmonic source and the two lateral ones, and $\Delta \phi_{\omega}=\phi_{\omega}(I)-\phi_{\omega}\left(\alpha_{\omega_{1}} I\right)$ is the phase shift

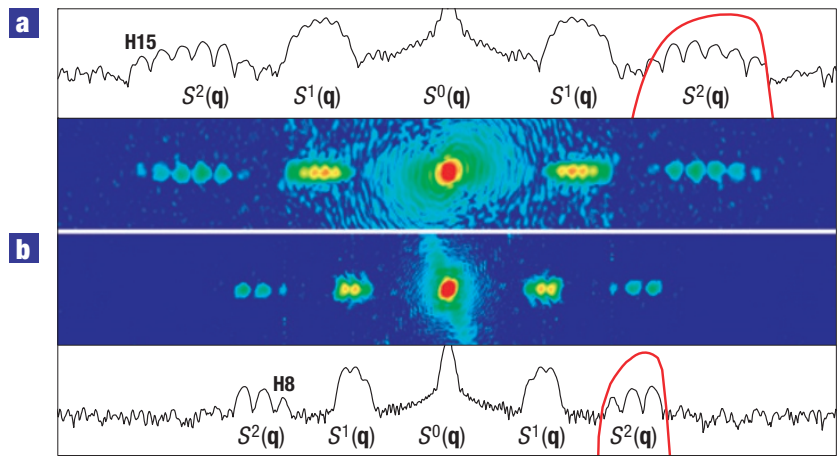

Figure 2 Spectra $\boldsymbol{S}(\boldsymbol{q})$ of two single-shot interferograms (in log scale). a, Case where harmonics 10-15 are selected using a 200-nm-thick tin filter. $\mathbf{b}$, Case where harmonics $8-10$ are selected with a $200-n m$-thick indium filter. The transmission curves of these filters are shown as red lines around the $S^{2}(\mathbf{q})$ term. In both cases, the generation is based on CWE on a silica target, and $\alpha_{\omega_{\perp}}=0.57$.

between these sources for frequency $\omega$ (where $\omega_{\mathrm{L}}$ is the driving laser frequency, and $I$ and $\alpha_{\omega_{\mathrm{L}}} I$ are the peak laser intensities for the central and lateral spots, respectively). No phase shift occurs between the two lateral harmonic sources, as they are generated at the same laser intensity. Using this simple formula, perfect fits of the experimental data can be obtained, as shown in Fig. 1c.

Equation (1) shows that the interference pattern contains two sets of fringes for each $\omega$, with spatial frequencies $\left|\mathbf{k}_{\omega}\right|$ and $2\left|\mathbf{k}_{\omega}\right|$. Fringes of frequency $\left|\mathbf{k}_{\omega}\right|$ correspond to the interference of the central harmonic source with either of the two lateral ones, whereas those of frequency $2\left|\mathbf{k}_{\omega}\right|$ correspond to the interference between the two outer sources. In usual two-source interferometry, phase terms are encoded in shifts of the fringes. Because of the symmetry of the present configuration with three sources, the phase shift $\Delta \phi_{\omega}$ affects the contrast of the $\left|\mathbf{k}_{\omega}\right|$ fringes, but not their positions.

To further analyse these interferograms, we calculate the Fourier transform $S(\mathbf{q})$ of $I(\mathbf{r})$ with respect to $\mathbf{r}$

$$
\begin{gathered}
S(\mathbf{q})=\int \mathrm{d} \omega I_{\omega}\left[S_{\omega}^{0}(\mathbf{q})+S_{\omega}^{1}(\mathbf{q})+S_{\omega}^{2}(\mathbf{q})\right] \\
S_{\omega}^{0}(\mathbf{q})=\left(1+2 \alpha_{\omega}\right) \tilde{F}_{\omega}(\mathbf{q}) \\
S_{\omega}^{1}(\mathbf{q})=2 \sqrt{\alpha_{\omega}} \cos \left(\Delta \phi_{\omega}\right)\left[\tilde{F}_{\omega}\left(\mathbf{q}-\mathbf{k}_{\omega}\right)+\tilde{F}_{\omega}\left(\mathbf{q}+\mathbf{k}_{\omega}\right)\right] \\
S_{\omega}^{2}(\mathbf{q})=\alpha_{\omega}\left[\tilde{F}_{\omega}\left(\mathbf{q}-2 \mathbf{k}_{\omega}\right)+\tilde{F}_{\omega}\left(\mathbf{q}+2 \mathbf{k}_{\omega}\right)\right],
\end{gathered}
$$

where $\tilde{F}_{\omega}(\mathbf{q})$ is the spatial Fourier transform of $F_{\omega}(\mathbf{r})$, whereas $S^{1}(\mathbf{q})$ and $S_{\omega}^{2}(\mathbf{q})$ are the Fourier transforms of the two sets of fringes discussed previously.

The total signal is the incoherent superposition of the interferograms corresponding to each frequency $\omega$ (equations (1) and (2)). As each frequency leads to a different fringe spacing, the $S_{\omega}^{1}(\mathbf{q})$ or $S_{\omega}^{2}(\mathbf{q})$ terms in the Fourier transform of the interferogram provide the extreme-ultraviolet spectrum $I_{\omega}$, convoluted with $\tilde{F}_{\omega}(\mathbf{q})$. This set-up thus constitutes a remarkably simple and cheap single-shot Fourier spectrometer in the extreme-ultraviolet region. This is shown in Fig. 2, where two filters have been used to select different groups of harmonics. Its spectral resolution depends on the harmonic beam profile through $\tilde{F}_{\omega}(\mathbf{q})$, and increases with the divergence of this beam.

Equation (2) also shows that the phase shift $\Delta \phi_{\omega}$ between the central and lateral sources is encoded in the magnitude of the $S_{\omega}^{1}(\mathbf{q})$ 


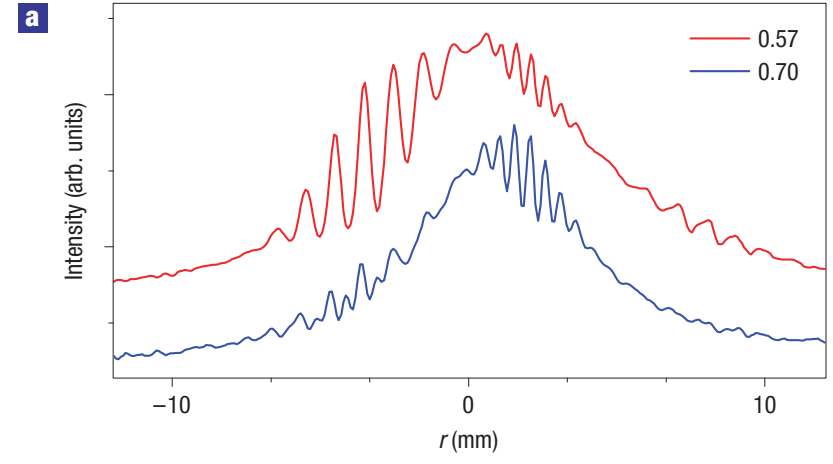

b

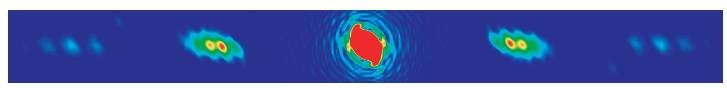

$\mathbf{6}$

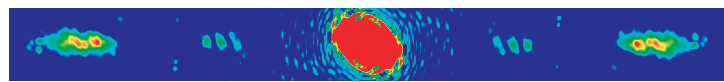

Figure 3 Effect of the phase shift $\Delta \phi_{\omega}$ on the interferogram. a, Lineouts of two single-shot interferograms, for two intensity ratios $\alpha_{\omega_{\mathrm{L}}}$ of 0.57 and 0.7 (corresponding to slit widths of the grating of respectively $4 \mathrm{~mm}$ and $3.2 \mathrm{~mm}$ ). The fringe pattern is different from the one shown in Fig. 1c, because of the larger numerical aperture $(f / 12$ instead of $f / 17)$ of the laser beam, which leads to a larger divergence of the harmonic beam and hence a wider interference field. b,c, Fourier transforms $S(\mathbf{q})$ of the corresponding interferograms (on a linear scale), showing the large change in relative weight of the $S_{\omega}^{1}(\mathbf{q})$ and $S_{\omega}^{2}(\mathbf{q})$ terms when $\Delta \phi_{\omega}$ goes from $\approx \pi\left(\mathbf{b}, \alpha_{\omega_{\mathrm{L}}}=0.57\right)$ to $\approx \pi / 2\left(\mathbf{c}, \alpha_{\omega_{\mathrm{L}}}=0.7\right)$.

term in the spectrum $S(\mathbf{q})$. To illustrate the influence of $\Delta \phi_{\omega}$, we have varied its value by changing $\alpha_{\omega_{\mathrm{L}}}$, the intensity ratio for the driving laser (Fig. 3). This is achieved by changing the width $l$ of the slits in the transmission grating. The case where $\Delta \phi_{\omega} \approx \pi / 2$ leads to particularly striking effects on the interferogram. In this case, the $S_{\omega}^{1}\left(\mathbf{k}_{\omega}\right)$ term in $S(\mathbf{q})$ is close to zero and fringes of frequency $\left|\mathbf{k}_{\omega}\right|$, which usually dominate the interferogram, now almost completely disappear, whereas the $2\left|\mathbf{k}_{\omega}\right|$ fringes keep a good contrast. We extract $\Delta \phi_{\omega}$ from the spectrum $S(\mathbf{q})$ (see the Methods section and Supplementary Information) using the ratio:

$$
\begin{aligned}
R_{\omega} & =S\left(\mathbf{k}_{\omega}\right) / S\left(2 \mathbf{k}_{\omega}\right) \\
& \approx S_{\omega}^{1}\left(\mathbf{k}_{\omega}\right) / S_{\omega}^{2}\left(2 \mathbf{k}_{\omega}\right)=2 \cos \left(\Delta \phi_{\omega}\right) / \sqrt{\alpha_{\omega}} .
\end{aligned}
$$

The results of this measurement are shown in Fig. 4, for harmonics 11, 12 and 13, and for different intensity ratios $\alpha_{\omega_{\mathrm{L}}}$ corresponding to different $l$.

$\Delta \phi_{\omega}$ decreases from $\approx \pi$ to slightly less than $\pi / 2$ as the laser intensity ratio $\alpha_{\omega_{\mathrm{L}}}$ goes from about 0.57 to 0.74 . For each $\alpha_{\omega_{\mathrm{L}}}$, the phase shift $\Delta \phi_{\omega}$ between the central harmonic source and the two lateral ones is observed to vary linearly with harmonic frequency $\omega$ (Fig. 4a). A linear phase shift $\Delta \phi_{\omega}$ corresponds to a simple translation by $\tau=\Delta \phi_{\omega} / \omega$ in the time domain. In other words, these results show that the light fields $E^{0}(t)$ and $E^{1}(t)$, corresponding to the superposition of harmonics 11-13, emitted respectively by the central and lateral sources, are identical and simply shifted in time by a delay $\tau$, that is, $E^{0}(t)=E^{1}(t+\tau)$. Figure $4 \mathrm{~b}$ shows that $\tau$ decreases as intensity ratio $\alpha_{\omega_{\mathrm{L}}}$ gets closer to 1 , as expected. These delays are smaller than 100 as: this remarkable sensitivity of the method comes from the fact that the contrast of the $\left|\mathbf{k}_{\omega}\right|$ fringes goes from maximum to zero for a change of only $\pi / 2$ of the phase of harmonic $n$ (Fig. 3 ), corresponding to a
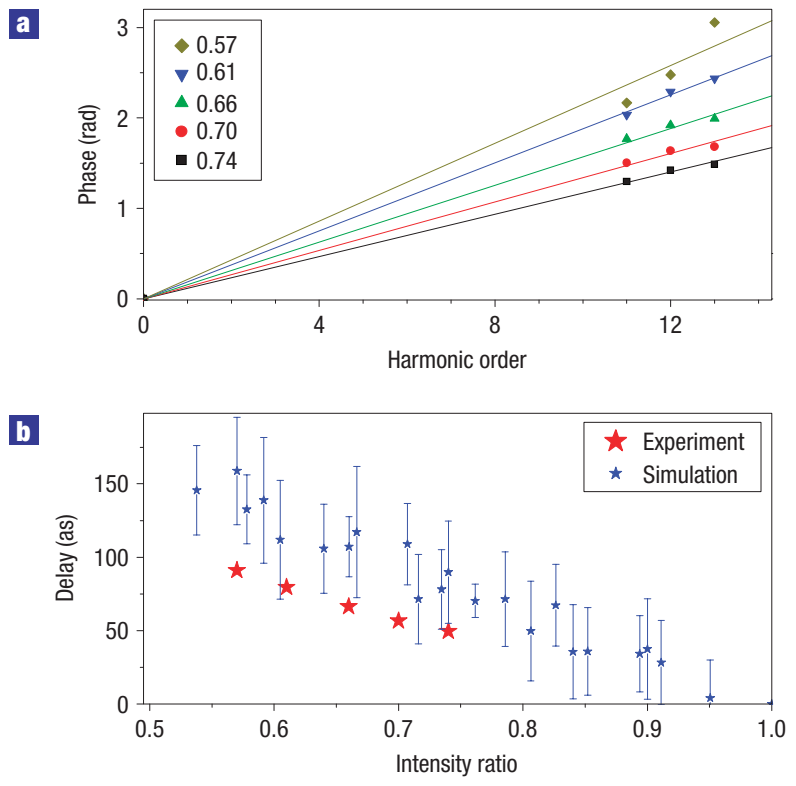

Figure 4 Relative phases $\Delta \phi_{\omega}$ between the central and lateral sources. a, Phase shift plotted as a function of the harmonic order (for orders 11-13), for different laser intensity ratios $\alpha_{\omega_{L}}$, the values of which are given in the legend. Each point is an average over five laser shots, and has an uncertainty of $\approx 10 \%$. The lines are linear fits, $\Delta \phi_{\omega}=\omega \tau$. $\mathbf{b}$, The delay $\tau$ deduced from these data as a function of $\alpha_{\omega_{L}}$. These measurements are compared with the results of PIC simulations carried out at different laser intensities. In this case, the error bars correspond to the fluctuations in delay between pairs of attosecond pulses in the two trains (see the Supplementary Information).

shift in time of $T_{\mathrm{L}} / 4 n$, with $n \approx 10$ and $T_{\mathrm{L}}=2.6$ fs for an $800 \mathrm{~nm}$ laser field.

Numerical simulations with particle-in-cell (PIC) codes provide a physical interpretation of this delay $\tau$ (ref. 20). In CWE, trains of attosecond pulses are emitted by collective electron oscillations at the plasma-vacuum interface, which are triggered once every optical cycle in the wake of electron bunches ${ }^{12}$. These electron bunches are formed as the electric field pulls some electrons into vacuum, and then, when it changes sign, pushes them back into the overdense plasma ${ }^{22,23}$. When the peak intensity of the laser pulse is changed, the return time of these so-called 'Brunel electrons' to the plasma changes by a fraction of the laser optical period. The same time shift is transferred to the attosecond pulses. According to simulations, this is the main effect of an intensity variation of the driving laser on the attosecond pulse train.

The delay associated with this effect, obtained from one-dimensional PIC simulations, is shown in Fig. 4, as a function of the intensity ratio $\alpha_{\omega_{\mathrm{L}}}$. It differs from the experimental values by a factor 1.6, but the evolutions with $\alpha_{\omega_{\mathrm{L}}}$ are similar. This qualitative agreement is satisfactory, as a PIC code is only a model of the actual plasma, which does not take all physical processes into account (for example, collisions). For instance, whereas the expansion of the plasma is only approximately reproduced by PIC simulations, the delay $\tau$ depends on the length and shape of the density gradient at the plasma-vacuum interface. This comparison with simulations shows that our measurements give access to the change in return time of Brunel electrons with laser intensity, with sublaser cycle temporal resolution.

In the past 15 years, HHG from gases has been instrumental in providing a comprehensive understanding of the interaction 
of intense lasers with atoms and molecules ${ }^{3,4}$. Our measurements show that HHG from plasma mirrors can be used in a similar way as a probe of the coherent dynamics of plasmas driven by high-intensity lasers. Fully exploiting this new probe will require measurements of the harmonic field with attosecond resolution. Attosecond pulses can be generated not only when electrons return to the plasma (CWE), but also when they escape towards vacuum and reach relativistic velocities, thus inducing a Doppler upshift of the reflected light ${ }^{11,24,25}$. Measurements of the attosecond pulse trains associated with these two processes, and of their timing with respect to the driving laser optical cycle, should thus provide direct insight into the different stages of sublaser cycle electron dynamics. Studying this dynamics is essential for a proper understanding of many phenomena in laser-plasma physics, such as ion or electron acceleration, or short-wavelength-light generation, either by HHG or other mechanisms such as $\mathrm{K} \alpha$ emission $^{26}$. From the point of view of applications, demonstrating the coherent character of HHG from plasma mirrors, as we have done here for CWE, is an important step for the development of this ultrashort-light source. Obtaining extremely short wavelengths will require the use of the Doppler effect occurring at higher intensities. Although this remains to be established experimentally, the coherence of the light beam is also expected to be preserved in this process ${ }^{20}$.

\section{METHODS}

\section{EXPERIMENTAL SET-UP}

We use $60 \mathrm{fs}, 10 \mathrm{TW}$ pulses delivered by a chirped-pulse-amplification titanium-sapphire laser. A double-plasma-mirror optical switch is used at the output of the laser chain to improve the temporal contrast ratio by four orders of magnitude ${ }^{27}$. A transmission grating, consisting of a frosted-glass comb of $1 \mathrm{~cm}$ periodicity, with $4-\mathrm{mm}$-wide slits, is placed into the beam. To vary the effective slit width, a second identical grating is placed behind the first one and translated horizontally. This beam is then focused with an off-axis parabolic mirror of $500 \mathrm{~mm}$ focal length, with a numerical aperture that can be varied from $f / 10$ to $f / 25$ (corresponding to beam diameters of 50-20 mm). Diffraction from this grating leads, at the laser focus, to three main focal spots separated by $a=40 \mu \mathrm{m}$. For a slit width of $4 \mathrm{~mm}$, the intensity ratio between the central spot and the lateral ones is 0.57 .

Harmonics of the $800 \mathrm{~nm}$ incident laser field are generated on reflection on a bulk silica target at $45^{\circ}$ incidence, by the CWE mechanism ${ }^{12} .15 \mathrm{~cm}$ away from the target, the beam reflects on two plates with an anti-reflection coating at the laser wavelength (not shown in Fig. 1a). This eliminates most of the fundamental light. Different groups of harmonics can then be selected using filters. The resulting extreme-ultraviolet beam is detected at $D=38 \mathrm{~cm}$ from the source, using double-stage microchannel plates coupled to a phosphor screen, the fluorescence of which is imaged on a CCD (charge-coupled device) camera. The total spatial resolution of the set-up is estimated to be $90 \mu \mathrm{m}$, which is high enough to properly resolve the fringe pattern of a few hundred microns periodicity.

\section{DATA ANALYSIS}

From the interferograms, we calculate $\cos \left(\Delta \phi_{\omega}\right)$ using the ratio $R_{\omega}$ defined in equation (3). This requires knowledge of the intensity ratio $\alpha_{\omega}$ (equation (3)). To this end, we have measured the dependence of the harmonic signal on the laser intensity (see Supplementary Information, Fig. S1), and use these data to deduce $\alpha_{\omega}$ from $\alpha_{\omega_{\mathrm{L}}}$, the intensity ratio for the incident laser field, which is easily and accurately calculated from the characteristics of the grating using diffraction theory. The value of $\cos \left(\Delta \phi_{\omega}\right)$ provides $\left|\Delta \phi_{\omega}+2 n \pi\right|$. The sign of $\Delta \phi_{\omega}$ is determined from numerical simulations, and the value of $n$ is determined from the measured divergence of the harmonic beam (see Fig. 1c and Supplementary Information).

\section{PIC SIMULATIONS}

We use the one-dimensional PIC code EUTERPE (ref. 28). The results of Fig. 4 were obtained from simulations where both ions and electrons are mobile. We start with an infinitely steep plasma-vacuum interface, and initial ion and electron temperatures of 0.1 and $0.5 \mathrm{keV}$, respectively. Collisions are not taken into account. The plasma density is $110 n_{\mathrm{c}}$. The maximum intensity that we used is $I \lambda^{2}=1.2 \times 10^{17} \mathrm{~W} \mathrm{~cm}^{-2} \mu \mathrm{m}^{-2}$, and the intensities for the other simulations were deduced from this value using the ratio $\alpha_{\omega_{\mathrm{L}}}$. We filter a group of harmonics in the spectrum of the pulse reflected from the plasma, to obtain a train of attosecond pulses in the time domain, and then measure the temporal position of the peak of each of these attosecond pulses. The data in Fig. 4 correspond to the average value of the delay between attosecond pulses in two trains generated at two different laser intensities.

Received 13 February 2008; accepted 8 May 2008; published 8 June 2008.

\section{References}

1. Chapman, H. N. et al. Femtosecond time-delay X-ray holography. Nature 448, 676-679 (2007).

2. Cavalieri, A. L. et al. Attosecond spectroscopy in condensed matter. Nature 449, 1029-1032 (2007).

3. Corkum, P.B. \& Krausz, F. Attosecond science. Nature Phys. 3, 381-387 (2007).

4. Agostini, P. \& DiMauro, L. The physics of attosecond pulses. Rep. Prog. Phys. 67, 813-855 (2004).

5. Dromey, B. et al. High harmonic generation in the relativistic limit. Nature Phys. 2, 456-459 (2006).

6. Tsakiris, G., Eidmann, K., Meyer-ter-Vehn, J. \& Krausz, F. Route to intense single attosecond pulses. New J. Phys. 8, 19 (2006).

7. Murnane, M. M., Kapteyn, H. C., Gordon, S.P. \& Falcone, R.W. Ultrashort X-ray pulses. Appl. Phys. B 58, 261-266 (1994).

8. Chapman, H. N. et al. Femtosecond diffractive imaging with a soft-X-ray free-electron laser. Nature Phys. 2, 839-843 (2006).

9. Le Deroff, L. et al. Measurement of the degree of spatial coherence of high-order harmonics using a Fresnel-mirror interferometer. Phys. Rev. A 61, 043802 (2000).

10. Zhang, J. et al. Coherence and bandwidth measurements of harmonics generated from solid surfaces irradiated by intense picosecond laser pulses. Phys. Rev. A 54, 1597-1603 (1996).

11. Thaury, C. et al. Plasma mirrors for ultrahigh intensity optics. Nature Phys. 3, 424-429 (2007).

12. Quéré, F. et al. Coherent wake emission of high-order harmonics from overdense plasmas. Phys. Rev. Lett. 96, 125004 (2006)

13. Collett, E. \& Wolf, E. Is complete spatial coherence necessary for the generation of highly directional light beams? Opt. Lett. 2, 27-29 (1978).

14. Salières, P. et al. Frequency-domain interferometry in the XUV with high-order harmonics. Phys. Rev. Lett. 83, 5483-5486 (1999).

15. Cavalieri, S. et al. Ramsey-type spectroscopy with high-order harmonics. Phys. Rev. Lett. 89, 133002 (2002).

16. Gordienko, S., Pukhov, A., Shorokhov, O. \& Baeva, T. Relativistic Doppler effect: Universal spectra and zeptosecond pulses. Phys. Rev. Lett. 93, 115002 (2004).

17. Dromey, B. et al. Bright multi-keV harmonic generation from relativistically oscillating plasma surfaces. Phys. Rev. Lett. 99, 085001 (2007).

18. Lewenstein, M., Salières, P. \& L'Huillier, A. Phase of the atomic polarization in high-order harmonic generation. Phys. Rev. A 52, 4747-4754 (1995)

19. Corsi, C. et al. Direct interferometric measurement of the atomic dipole phase in high-order harmonic generation. Phys. Rev. Lett. 97, 023901 (2006).

20. Quéré, F. et al. Phase properties of high-order harmonics reflected from plasma mirrors. Phys. Rev. Lett. 100, 095004 (2008).

21. Zerne, R. et al. Phase-locked high-order harmonic sources. Phys. Rev. Lett. 79, 1006-1009 (1997).

22. Brunel, F. Not-so-resonant, resonant absorption. Phys. Rev. Lett. 59, 52-55 (1987).

23. Bonnaud, G., Gibbon, P., Kindel, J. \& Williams, E. Laser interaction with a sharp-edged overdense plasma. Laser Part. Beams 9, 339-354 (1991).

24. Bulanov, S. V., Naumova, N. M. \& Pegoraro, F. Interaction of an ultrashort, relativistically strong laser-pulse with an overdense plasma. Phys. Plasmas 1, 745-757 (1994).

25. Lichters, R., Meyer-ter-Vehn, J. \& Pukhov, A. Short-pulse laser harmonics from oscillating plasma surfaces driven at relativistic intensity. Phys. Plasmas 3, 3425-3437 (1996).

26. Rischel, C. et al. Femtosecond time-resolved X-ray diffraction from laser-heated organic films. Nature 390, 490-492 (1997)

27. Lévy, A. et al. Double plasma mirror for ultrahigh temporal contrast ultraintense laser pulses. Opt. Lett. 32, 310-312 (2007)

28. Bonnaud, G. \& Reisse, C. Particle code study of the influence of non-monochromaticity of laser light on stimulated Raman scattering in laser-irradiated plasmas. Nucl. Fusion 26, 633-646 (1986).

Supplementary Information accompanies this paper on www.nature.com/naturephysics.

\section{Acknowledgements}

Financial support from Agence Nationale pour la Recherche, through program BLAN06 - 3_134072 (ILAR), is gratefully acknowledged.

\section{Author contributions}

Idea and experiment design by F.Q. and C.T.; measurements by C.T., H.G, R.L. and F.Q.; data analysis by C.T., F.Q. and J.-P.G.; P.M. and Ph.M. contributed to scientific discussions and support; paper written by F.Q., C.T. and H.G.

\section{Author information}

Reprints and permission information is available online at http://npg.nature.com/reprintsandpermissions Correspondence and requests for materials should be addressed to F.Q. 